Case Report

\title{
Development of Some Organs Derived from the Three Embryonic Germ Layer in a Degus Ectopic Pregnancy and Presence of a Cytotrophoblast That Mimics Human Chorionic Placenta
}

\author{
C. Bosco, E. Díaz, J. González, and R. Gutierrez \\ Placenta and Fetal Development Laboratory, Anatomy and Developmental Biology Programme, Institute of Biomedical Sciences, \\ Faculty of Medicine, University of Chile, Independencia 1027, Casilla Postal 70079, 8380453 Santiago, Chile \\ Correspondence should be addressed to C. Bosco; cbosco@med.uchile.cl
}

Received 11 April 2014; Accepted 2 July 2014; Published 16 July 2014

Academic Editor: Isabel Pires

Copyright (c) 2014 C. Bosco et al. This is an open access article distributed under the Creative Commons Attribution License, which permits unrestricted use, distribution, and reproduction in any medium, provided the original work is properly cited.

\begin{abstract}
This report describes a case of abdominal pregnancy in an adult female degu from which we recovered two large tissular masses from the peritoneal cavity. The bigger one showed a number of thin vascular connections to the serosa layer of the small intestine. It was also directly connected to the smaller mass by a thin membranous process. The surface of the bigger mass facing the small intestine wall showed the presence of chorionic villous that resembled a villous human chorionic placenta, rather than the hemomonochorial labyrinthine placenta, characteristic of this species. This unusual finding leads us to postulate that in the degu's uterus the cytotrophoblast is exposed to a number of factors that will activate cascades of cellular and molecular events that ultimately will be signaling the cytotrophoblast to develop into a labyrinthine hemomonochorial placenta. In absence of the proper uterine environment, as is the case of the abdominal pregnancy in the peritoneal cavity reported here, the lack of signaling will lead the cytotrophoblast to develop into a villous chorionic placenta, similar to that observed in human.
\end{abstract}

\section{Introduction}

No uniform criterion for human or animal ectopic pregnancy classification exists. In order to facilitate a more didactic approach for the study of ectopic pregnancy, various criteria have been taken into consideration. Ectopic or extrauterine pregnancy denotes a pregnancy or an implantation event that occurs elsewhere rather than within the uterine cavity [1]. Although ectopic pregnancy has been reported in several species, its occurrence is considered a low incidence process [2].

Two main types of ectopic pregnancy have been described: (i) tubal and (ii) abdominal pregnancy $[2,3]$. It is known that approximately $1.3 \%$ of the ectopic pregnancies are abdominal [4], and they occur through direct implantation into the peritoneal surface. This type of ectopic pregnancy is further divided into primary and secondary types. Primary abdominal pregnancy involves the extrusion of the ovum from the fimbriated end of the uterine tube into the peritoneal cavity, either after or possibly before fertilization [2], followed by its implantation and ulterior development within the abdominal cavity. The implantation has been described to occur in a variety of extrapelvic organs such as omentum, liver, spleen, and the walls of the small and large intestines $[1,5,6]$. Secondary abdominal pregnancy is usually a consequence of rupture of the uterus or oviducts and the release of the fertilized ovum or developing embryo into the abdominal cavity. In this case, the placentae and membranes may or may not be expelled from these organs [2].

\section{Case Report}

A four-year-old female Octodon degus, from the breeding colony maintained at the Anatomy and Developmental Biology Programme, Institute of Biomedical Sciences, Faculty of Medicine, University of Chile, was found dead overnight and was submitted to the diagnostic laboratory for necropsy. During the course of postmortem examination, two large tissue 


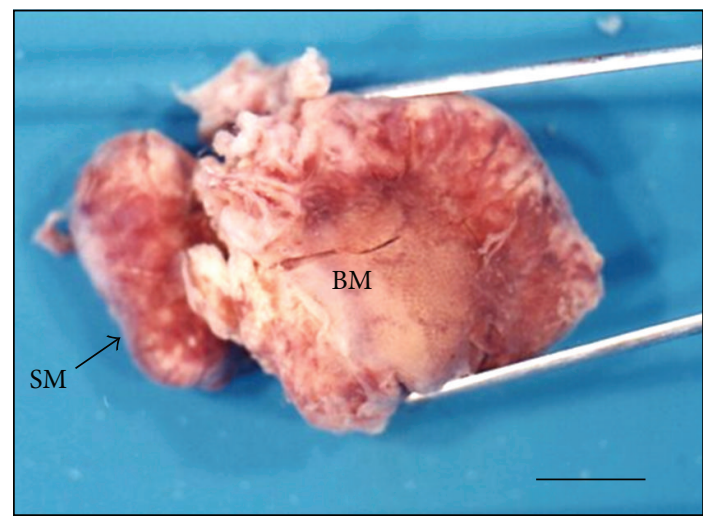

(a)

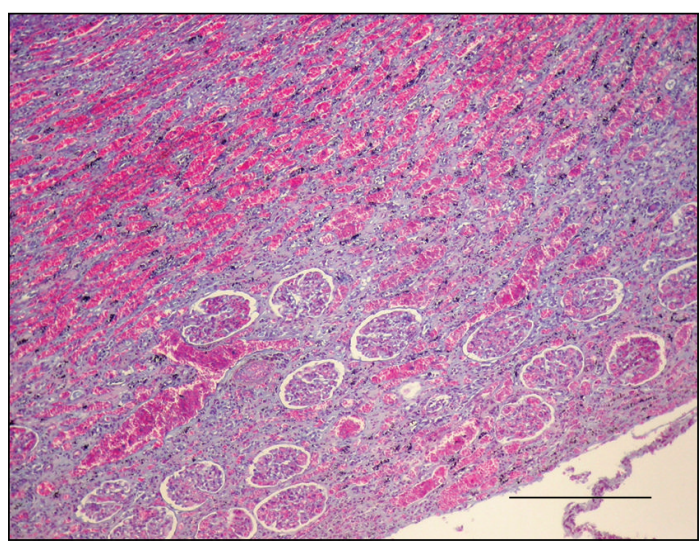

(c)

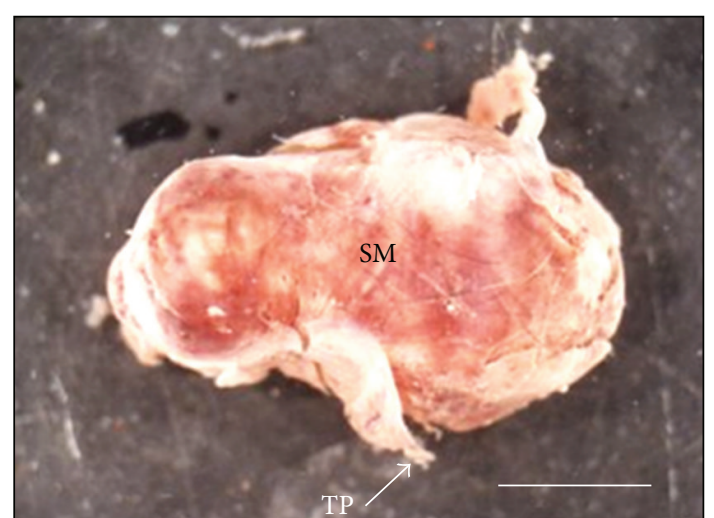

(b)

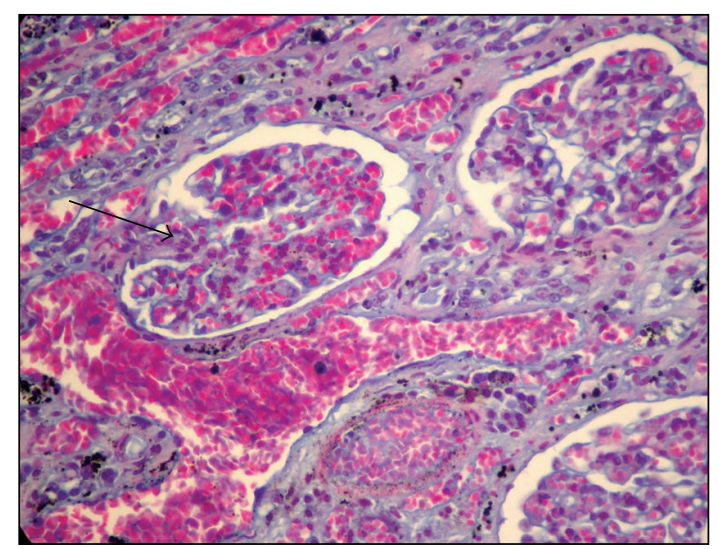

(d)

Figure 1: Macro- and microscopic views of the degu's intraperitoneal masses. (a) External aspect of the bigger (BM) and the smaller (SM) masses. They were enveloped by a thin translucent membrane and were profusely irrigated. The arrows depict the zone of the chorionic villus (CV) (see also Figure 5) and the zone of the apparent umbilical cord (UC) (see also Figure 3(d)). A remnant of the small intestine (SI) near the bigger mass is also observed. (b) Smaller mass showing the thin process (white arrow heads) that connects to the bigger mass. (c) Photomicrograph showing the kidney-like tissue formation towards the peripheral surface of the bigger mass. (d) Higher magnification of a selected region from (c) showing three glomerulus, one of them depicting a clear vascular pole (arrow). (c and d) Trichromic Masson staining. Calibration bars (a) $=1.5 \mathrm{~cm}$; (b) $=3.2 \mathrm{~cm}$; (c) $=198 \mu \mathrm{m}$; (d) $=52 \mu \mathrm{m}$.

masses were found and removed from the peritoneal cavity. There were no signs of hemorrhage, lesions, or anatomical abnormalities, and the remaining abdominal organs showed normal appearance. In this context, we postulated that these tissue masses could correspond to either a teratoma or an ectopic pregnancy and decided to perform further histological observations.

\section{Material and Methods}

The masses were carefully dissected, cut into $0,5 \mathrm{~cm}$ pieces, and immediately fixed by immersion into $10 \%$ buffered formalin solution for 24 hours. In order to assess the histological integrity of the tissues, other organs including heart, kidneys, brain, liver, spleen, oviducts, ovaries, and uterus were also dissected and fixed. All the samples were thoroughly dehydrated through graded alcohols before being embedded in paraffin wax.

Several sections $4 \mu \mathrm{m}$ thick were obtained from each sample and mounted onto gelatinized slides. Due to the embryonic constitution of the tissue, some difficulties aroused during the cutting process or the mounting of the sections.

Routine histological analysis was performed using hematoxylin-eosin (H/E), trichromic Masson-staining, and PAP techniques. All sections were observed and photographed under a Zeiss Axiolab light microscopy (Carl Zeiss, Germany).

\section{Results}

The two large tissular masses found in the degus peritoneal cavity are shown in Figures 1(a) and 1(b). They were round in shape, the bigger mass measuring $3 \times 3.5 \times 0.9 \mathrm{~cm}$ and the smaller one $3 \times 1.9 \times 0.9 \mathrm{~cm}$. Both masses were enveloped by a thin translucent membrane and were profusely irrigated. Under microscopic observation, the others studied organs showed no signs of lesions or degenerative changes that are usually associated to the process of autolysis.

The two masses were united between them by a thin process (see Figure 1(b)) and the bigger one was connected 


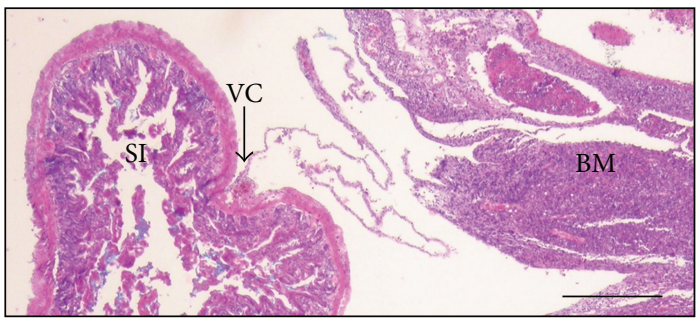

(a)

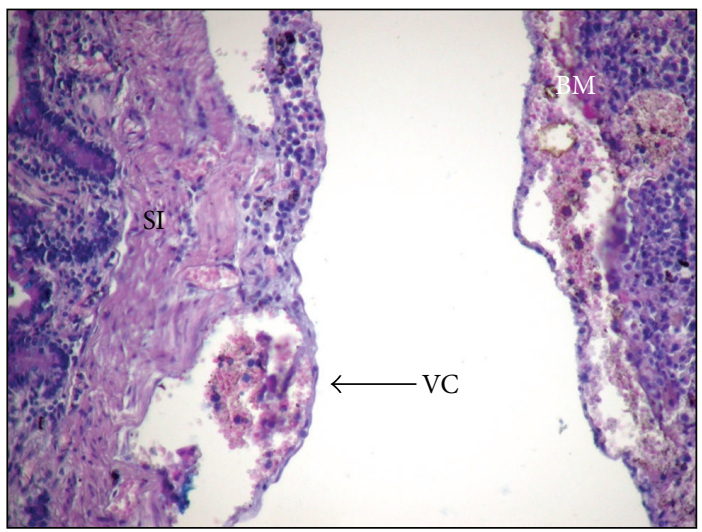

(c)

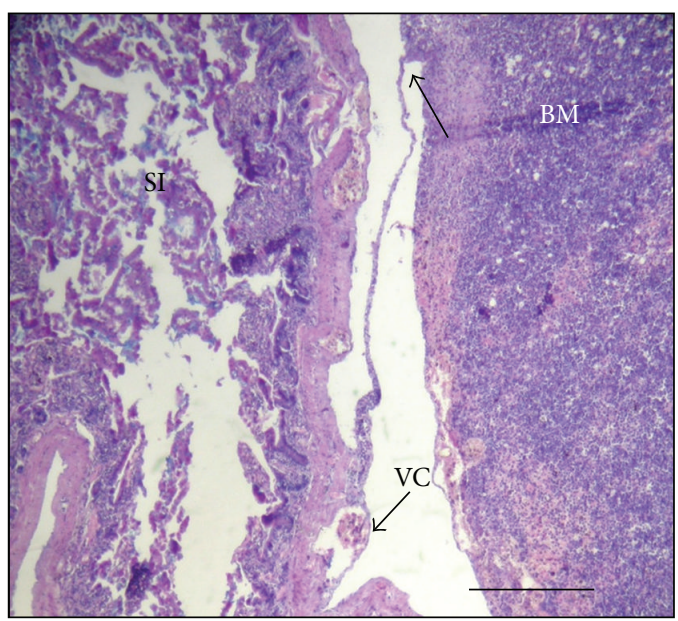

(b)

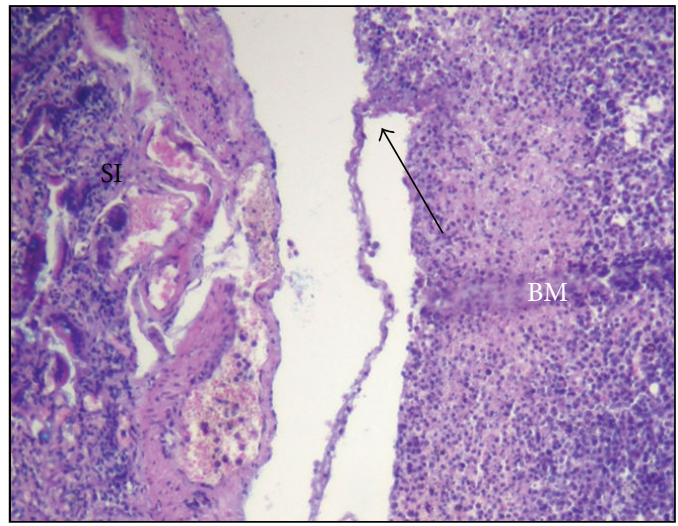

(d)

Figure 2: Vascular connections of the bigger mass to the small intestine. (a) Photomicrograph showing the vascular connection (VC, arrows) between the bigger mass (BM) and the serosa layer of the small intestine (SI). (b) Another vascular connection (arrows) between the same structures. H/E staining. Calibration bars: (a) $=223 \mu \mathrm{m}$; (b) $=223 \mu \mathrm{m}$.

to the serosa layer of the small intestine of the animal, as it was evidenced by microscopic observation (Figure 2).

Reproductive organs such as ovaries, oviducts, and both uterine horns displayed normal histological appearance (see Figures 3(a), 3(b), and 3(c)). In one of the ovaries, the presence of two functional corpora lutea was evident and they were surrounded by multiples degenerative corpora lutea (Figure 3(c)). The bicorneal uterus showed some traces of inflammatory secretion in the lumen (Figure 3(a)).

Inside both masses, areas of developing tissues were observed which showed characteristics of kidney (Figures $1(\mathrm{c})$ and $1(\mathrm{~d})$ ) and pancreas (Figure 4). Developing tissues showing characteristics of liver, striated muscle, bone, and suprarenal gland, as well as some fibrous tissue were also observed (not shown).

Histologically, the masses consisted of various tissues derived from all three embryonic germ layers, that is, ectoderm (nervous cells in the pancreas [7] and nerve fibers), mesoderm (kidney, striated muscle, and bone), and endoderm (glandular tissues such as liver and pancreas), but appendages of the skin were not found. Unexpectedly, we observed in the zone between the bigger mass and the small intestine the presence of some chorionic villus (Figure 5), whose aspect resembled that observed in the human placenta. Briefly, each villus consisted of a central core of loose connective tissue surrounded by a double layer of trophoblast, an outer syncytiotrophoblast and an inner cytotrophoblast. The core contained the fetal placental capillaries (Figures 5(b) and 5(d)) [8]. It must be emphasized that, in the degu's placental barrier, the fetal capillaries are separated from the maternal blood by a single layer of labyrinthine fine syncytium, derived from the cytotrophoblast of the subplacenta $[9,10]$. In addition, a structure resembling an umbilical cord was observed inserted into the bigger mass (Figure 3(d)).

\section{Discussion}

This is the first time that the occurrence of an ectopic pregnancy in the degu is reported, although a direct placental attachment to the maternal viscera was not observed, probably due to scarse development of the subplacenta (cytotrophoblast) which disappear when it differentiates into 


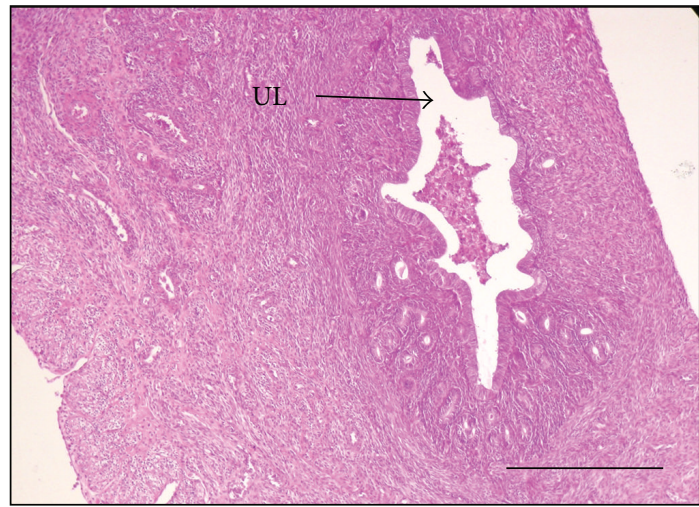

(a)

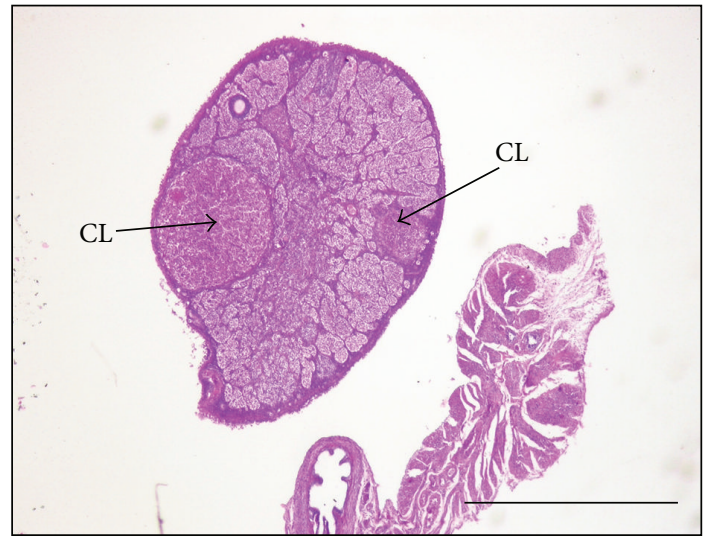

(c)

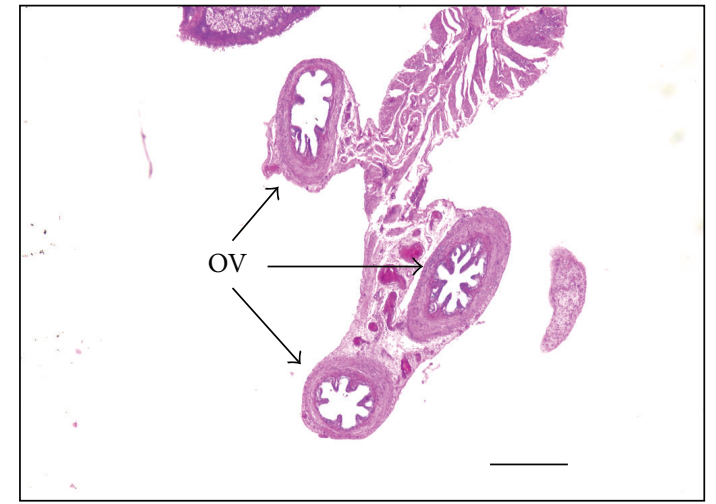

(b)

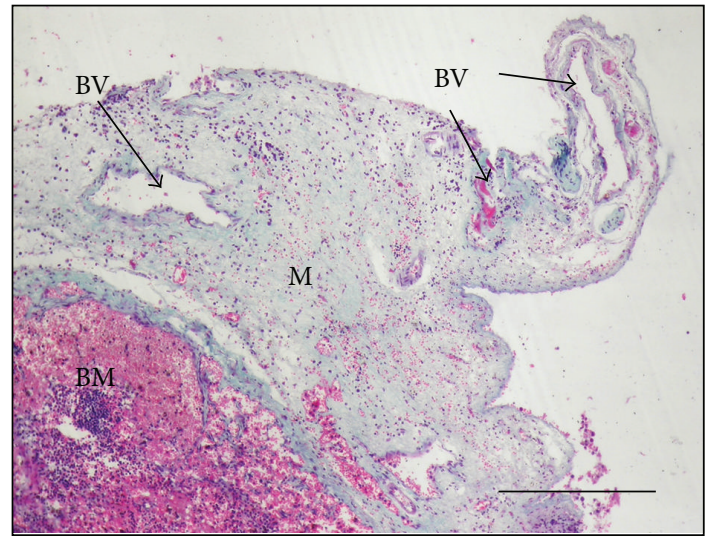

(d)

FIGURE 3: Normal aspect of the maternal reproductive organs. (a) The uterus evidences the endometrial, myometrial, and perimetrial layers. The lumen of the organ (UL) shows the presence of some type secretion. (b) Transversal sections of the oviduct (OV) displaying normal evaginations of the mucosa layer. (c) Transversal section of an ovary showing the presence of two functional corpora lutea (CL) surrounded by multiples degenerative corpora lutea. Figure 3(d) shows mesenchymal tissue (M) organized as a structure with large blood vessels (BV), with the appearance of an umbilical cord attached to the bigger mass (BM). (a, b, and c) H/E staining; (d) PAP staining; bars: (a) = 223 $\mu$ m; (b) $=245 \mu \mathrm{m} ;(\mathrm{c})=245 \mu \mathrm{m}$ and $(\mathrm{d})=223 \mu \mathrm{m}$.

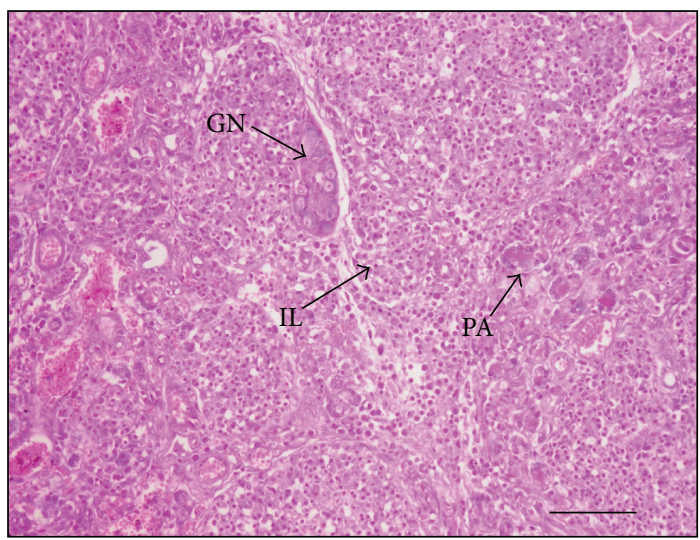

(a)

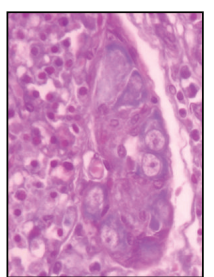

(b)

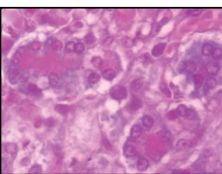

(c)

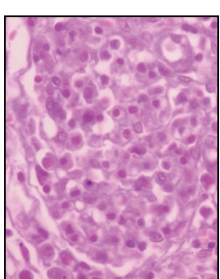

(d)

FIGURE 4: Developing pancreatic tissue in the bigger mass. (a) Low magnification microphotograph showing islets of Langerhans (IL) in the endocrine pancreatic tissue and pancreatic acini (PA) in the exocrine portion. A group of ganglionic neurons (GN) can also be observed. (b, c, and d) correspond to higher magnifications of the regions described in (a). H/E staining; bars (a) $=223 \mu \mathrm{m}$; (b) $=179 \mu \mathrm{m}$; (c) $=179 \mu \mathrm{m}$ : (d) $=179 \mu \mathrm{m}$. 


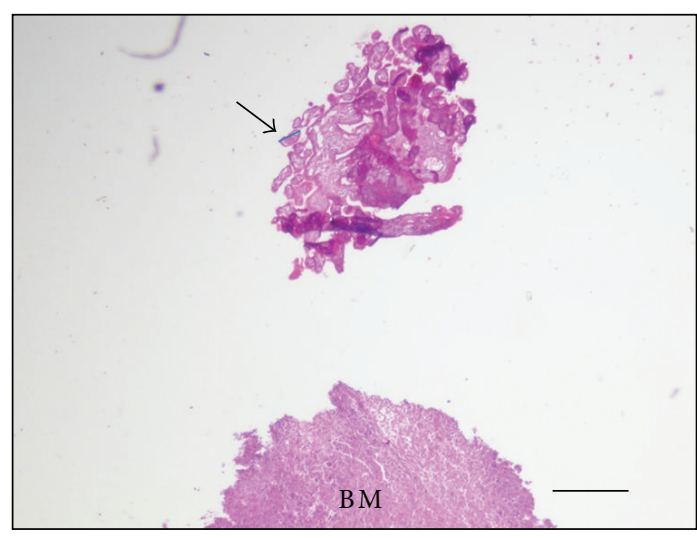

(a)

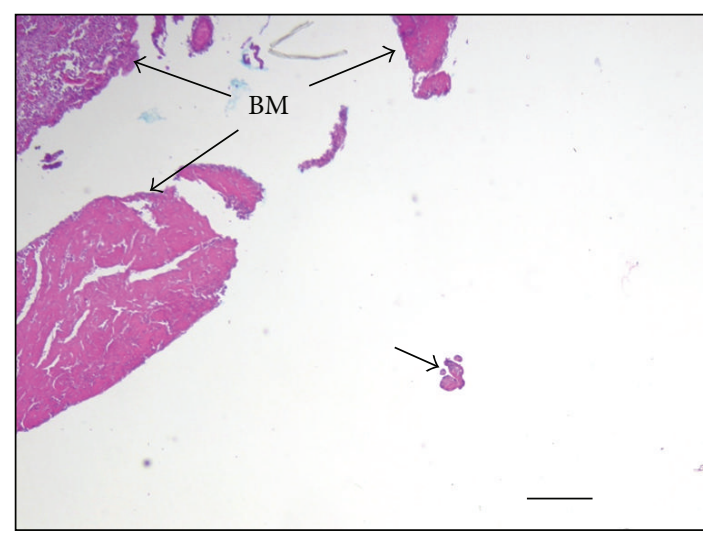

(c)

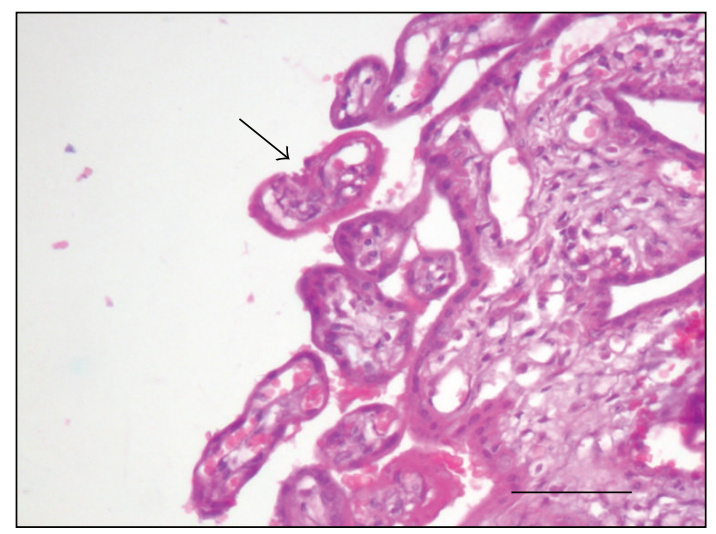

(b)

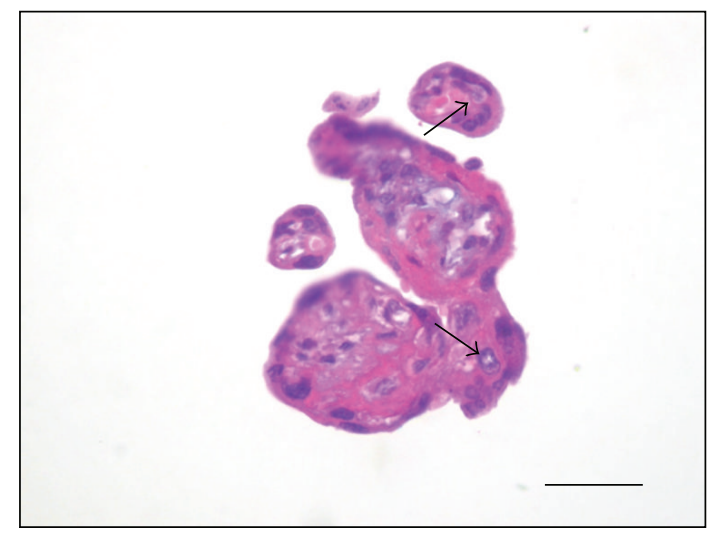

(d)

FIGURE 5: Chorionic villous-like structures observed in the vicinity of the bigger mass. (a) The bigger mass (BM) was found in relation to a number of chorionic villi (arrow) that at higher magnification resemble the human placenta. (b) High magnification of the chorionic villi showed in (a). These villi display a human placental appearance rather than degus. In fact, each villus has a central core of loose connective tissue surrounded by a double layer of trophoblast, an outer syncytiotrophoblast and an inner cytotrophoblast. Fetal placental capillaries in the central core are clearly observed. (c) Spatial relation of the bigger mass (BM) with another group of chorionic villi (CV) with the same human placental appearance as shown in ( $\mathrm{a}$ and b). (d) Higher magnification of the chorionic villi (arrow) observed in (c) showing the presence of two inner cytotrophoblasts (arrows). H/E staining. Calibration bars: (a) $=245 \mu \mathrm{m}$; (b) $=1225 \mu \mathrm{m}$; (c) $=245 \mu \mathrm{m}$; (d) $=600 \mu \mathrm{m}$.

syncytiotrophoblast [10]. Clinically, there were no pathognomonic signs or symptoms of the presence of these masses in the peritoneal cavity and the animal death was probably the result of compression of surrounding structures such as heart and lungs. There was no apparent antemortem abdominal inflammation, neither fluid accumulation nor necrosis associated to the tissues.

Histologically, the masses consisted of tissues derived from all three embryonic germ layers, but appendages of the skin were not observed. It is well known that teratomas are tumors composed of tissues derived from the three germinal layers of the embryo, but in addition appendages of the skin such as hairs, sebaceous gland, and sweat glands, often associated with dermoid tissue, are frequently presented in teratomas. They may occur either as a cystic lesion, usually benign, or as a solid tumor, usually malignant [11]. Thus, the lack of appendages of the skin and the presence of some villous placental tissue in the findings reported here support the notion of a primary ectopic pregnancy in the degus.

In a guinea pig (other caviomorph rodents) study about ectopic pregnancy, placental attachment to the left peritoneal wall was clearly identified, but unfortunately the authors failed to show or even describe a placental structure [12]. On the other hand, Buckley and Caine [13] showed in hamster that one out of four ectopic fetuses had a placenta attached to the abdominal surface of the diaphragm and that all the other three fetuses were lying free in the abdomen, with no associated placentae, as occurs in the smaller mass described in this study. The authors concluded that these cases of abdominal pregnancy were more likely to be of the secondary type, in which the placentae have been extruded from the uterus along with one fetus but not with the others. In the present study, we did not observe evidence of uterine rupture or remains of placental tissues in the uterine wall.

We showed that the bigger mass was attached to the serosa layer of the small intestine (Figures 4(a), 4(b), 4(c), and $4(\mathrm{~d})$ ) by a number of thin irrigated processes such as those observed by Norén and Lindblom [14] whom, in a human case of abdominal pregnancy, reported that only minimal vascular connection with the peritoneal surface was presented. These authors declared that this was the first report in the literature of an abdominal pregnancy without placental 
attachment to the maternal viscera. In our study, histological examination showed that there was no decidual-cell response of maternal stromal tissue in the small intestine and therefore the area of placental attachment could not be determined.

In the case reported here, only microscopic observations allowed us to demonstrate that between the bigger mass and the small intestine the degus' scarse chorionic villus has histological characteristics of human placenta.

It is important to note that the placenta of the degu is hemomonochorial and labyrinthine as in the guinea pig $[9,15]$ and that the degus cytotrophoblast develops from the subplacenta, from which the syncytial labyrinthine placental barrier is formed $[10,16]$. On the other hand, Bland and Donovan [17] were able to induce abdominal implantation in guinea pig by introducing fertilized eggs into the peritoneal cavity. These authors found that trophoblast was produced in all cases, but the nontrophoblastic component of these implants was restricted to a mesenchyme-like tissue. They concluded that the higher rate of success (four out of seven animals) in induced abdominal implantation in guinea pigs may have been due to the greater ability of the guinea pig blastocyst to penetrate the peritoneum. In a histological micrograph, these authors showed a subplacenta and invasive trophoblast in the spleen but not the development of a real placental organ as we report in the present study. In addition, Bosco et al. $[10,16]$ demonstrated that in the degu the cytotrophoblast is found only in the subplacenta and not in the labyrinthine placental barrier as it is known to occur in the human villous placental barrier. In the human species, each villus consists of a central core of loose connective tissue surrounded by a double layer of trophoblast, an outer syncytiotrophoblast and an inner cytotrophoblast that differentiate to syncytiotrophoblast [8], as is the case in this report (see Figures 5(a), 5(b), 5(c), and 5(d)).

The evidence showed here strongly suggests that in the degu the uterine environment provides the cytotrophoblast with exposure to a number of factors that will activate signaling cascades that play a role in determining the developing of the cytotrophoblast into a labyrinthine hemomonochorial placenta. In the absence of uterine environment, as in the case of the peritoneal cavity, the lack of signaling will lead to the development of a villous chorionic placenta with human appearance.

Based on these results, we consider that our case report may motivate molecular biologists to determine the DNA base sequence of human cytotrophoblast in order to compare it with that from the degu subplacenta and thereby ascertain if they may share a common sequence. This would give a stronger support to our proposal of the degu as an experimental animal model for the study of preeclampsia in the early stages of placentation $[10,16]$.

Finally, we want to emphasize that the discovery of an ectopic pregnancy in a species other than human is often an incidental finding, as animals may show or not clinical signs of fever, lethargy, or anorexia. We want to note that our unexpected discovery corresponds to a serendipity case that allows our minds to observe, act, and propose ideas.

\section{Conflict of Interests}

The authors declare that there is not conflict of interests regarding the publication of this paper.

\section{References}

[1] R. Varma, L. Mascarenhas, and D. James, "Successful outcome of advanced abdominal pregnancy with exclusive omental insertion," Ultrasound in Obstetrics and Gynecology, vol. 21, no. 2, pp. 192-194, 2003.

[2] J. M. Corpa, "Ectopic pregnancy in animals and humans," Reproduction, vol. 131, no. 4, pp. 631-640, 2006.

[3] C. D. Buergelt and K. Russell, "Ectopic pregnancy in a dog," Veterinary Medicine, vol. 99, no. 3, pp. 225-226, 2004.

[4] J. Bouyer, J. Coste, H. Fernandez, J. L. Pouly, and N. Job-Spira, "Sites of ectopic pregnancy: a 10 year population-based study of 1800 cases," Human Reproduction, vol. 17, no. 12, pp. 3224-3230, 2002.

[5] A. N. Kalof, B. Fuller, and M. Harmon, "Splenic pregnancy: a case report and review of the literature," Archives of Pathology \& Laboratory Medicine, vol. 128, no. 11, pp. el46-e148, 2004.

[6] M. A. Onan, A. B. Turp, A. Saltik, N. Akyurek, C. Taskiran, and O. Himmetoglu, "Primary omental pregnancy: case report," Human Reproduction, vol. 20, no. 3, pp. 807-809, 2005.

[7] C. Bosco, E. Díaz, R. Gutiérrez, J. González, and J. Pérez, "Ganglionar nervous cells and telocytes in the pancreas of Octodon degus: extra and intrapancreatic ganglionar cells and telocytes in the degus," Autonomic Neuroscience: Basic and Clinical, vol. 177, no. 2, pp. 224-230, 2013.

[8] C. Bosco, "Morphology of the capillaries in the alpha and beta zone of human term placenta: the relationship between capillary morphology and the trophoblastic layer," Medical Science Research, vol. 22, no. 2, pp. 115-117, 1994.

[9] C. Bosco, "Ultrastructure of the degu term placental barrier (Octodon degus): a labyrinthine hemomonochorial placental model," Medical Science Research, vol. 23, no. 1, pp. 15-18, 1997.

[10] C. Bosco, C. Buffet, M. A. Bello, R. Rodrigo, M. Gutierrez, and G. García, "Placentation in the degu (Octodon degus): analogies with extrasubplacental trophoblast and human extravillous trophoblast," Comparative Biochemistry and Physiology A: Molecular and Integrative Physiology, vol. 146, no. 4, pp. 475-485, 2007.

[11] A. Marcolongo, G. Divirgilio, G. Bettili et al., "Immature mesenteric teratoma in a male newborn infant: prenatal ultrasonographic diagnosis and surgical treatment," Prenatal Diagnosis, vol. 17, no. 7, pp. 686-688, 1997.

[12] C. C. Hong and M. L. Armstrong, "Ectopic pregnancy in 2 guinea-pigs," Laboratory Animals, vol. 12, no. 4, pp. 243-244, 1978.

[13] P. Buckley and A. Caine, "A high incidence of abdominal pregnancy in the Djungarian hamster (Phodopus sungorus)," Journal of Reproduction and Fertility, vol. 56, no. 2, pp. 679-682, 1979.

[14] H. Norén and B. Lindblom, "A unique case of abdominal pregnancy: what are the minimal requirements for placental contact with the maternal vascular bed?" American Journal of Obstetrics and Gynecology, vol. 155, no. 2, pp. 394-396, 1986.

[15] B. F. King and A. C. Enders, "The fine structure of the guinea pig visceral yolk sac placenta.," American Journal of Anatomy, vol. 127 , no. 4 , pp. $394-414,1970$. 
[16] C. Bosco and C. Buffet, "Immunohistochemical identification of the extravillous trophoblast during the placentation of the degu (Octodon degus)," Journal of Experimental Zoology B, vol. 310, no. 6, pp. 534-539, 2008.

[17] K. P. Bland and B. T. Donovan, "Experimental ectopic implantation of eggs and early embryos in guinea-pigs," Journal of Reproduction and Fertility, vol. 10, no. 2, pp. 189-196, 1965. 

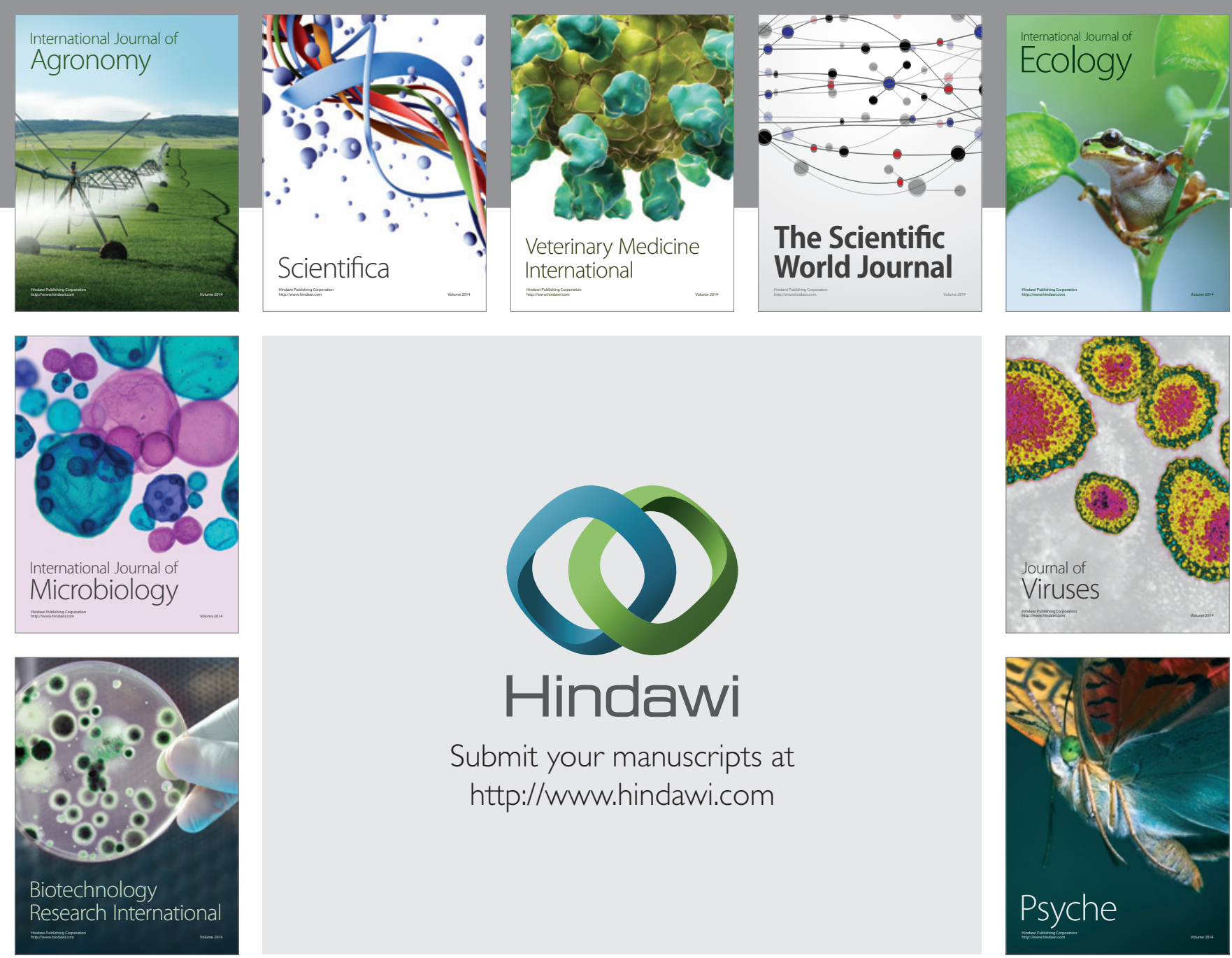

Submit your manuscripts at http://www.hindawi.com
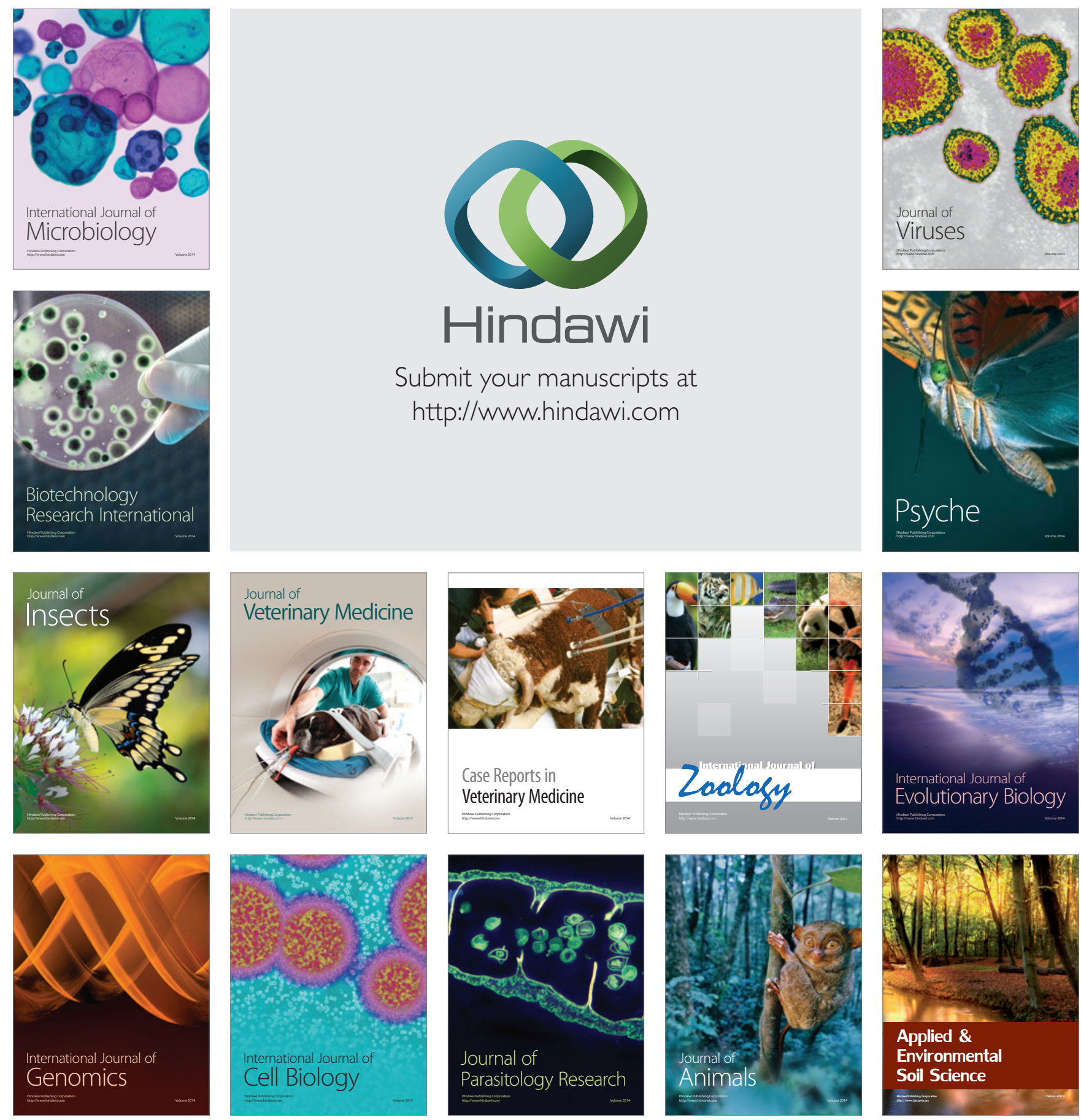